\title{
Emerging Approaches in Analysis and Evaluation in Sports Science
}

\author{
Ozdemir Galip* and Erogul Osman \\ Department of Biomedical Engineering, TOBB University of Economics \& Technology, Turkey
}

*Corresponding author: Ozdemir Galip, Department of Biomedical Engineering, TOBB University of Economics \& Technology, Turkey.

Received Date: September 05, 2018

Published Date: October 16, 2018

\begin{abstract}
It is not an easy task to analyze individual's performances, especially in team sports. There are numerous variables to be measured and considered to accurate analysis. Training, warm-up, motivation methods, strategies, procedures then can be arranged accordingly. Studies in sports sciences do not generally converge on a single global (optimum) solution, where instead various findings contribute for perpetual advances. In this review, research studies, recent approaches, and limitations are discussed. Moreover, future studies, trending approaches, and new performance analysis techniques are also discussed.
\end{abstract}

Keywords: Sports Science; Training, Stress; Gps; Physiological signals

\section{Introduction}

Quantifying the training and competitive match loads of players in sports can be performed in various ways not limited to kinematic, perceptual, psychological or physiological monitoring methods. These approaches provide supplementary data for trainers, however, the analysis procedure is generally challenging depending on the external conditions or individual differences between players or athletes. The collected raw data of these methods are large distinct datasets and they still cannot indicate a global solution or outcome for determining training load, injury risk/condition, performance evaluation. The main reason for this failure is the lack of quantifying the key aspects of human body movement with the difficulty of contact team sports. Rather than the traditional practitioner and trainer approaches, in recent years technological advances contributing to the analysis such as physiological recordings, video recordings and Global Position Systems as the most popular.

\section{Analysis and Evaluation Methods}

During the last decade, many research studies implemented to understand the effect of different mechanisms on performance.

\section{Effect of music}

There is a sharp increase in the number of studies which examined the relation between music and physical activity. Researchers have demonstrated the efficacy of music in aerobic performance, and positive effects of music on the athlete's performance [1-3]. On the contrary, other studies also reported no significant positive relation [4]. These studies focus on music exposure during warm-up and recovery as a motivation, arousal or relaxing tool $[2,3]$. There are no strong evidence indicating the relation between music type (instrumental or vocal, various beat per minutes value of the song etc.) and performance. Still, a detailed and well-designed study is necessary to unveil the effects of music on player performance.

\section{Warm-up routines}

The warm-up is implemented to increase muscle temperature that will yield as an increase in blood flow and optimized metabolic responses [5,6]. Optimized muscle temperature level will not only prevent fatigue but also improves training and competition performance [5,7]. Warm-up rouine strategies are popular research topics whereas many studies approve performance optimization is achieved [5-8]. Silva et. Al's [9] review show that in real-world application of warm-up strategies is dependent on individual experiences rather than scientific findings [6,8]. In team sports games, players should be able to do explosive efforts in short period of time as jumps, tackles, sprints having a high amount of velocity and direction changes $[10,11]$. Silva also reported nineteen articles for strategies for warm-up, up to 60 combinations of warm-ups. These studies compared the applied warm-up routines as follows: 
1. Warm-Up Structure (Volume, Intensity, Tasks)

2. Post-Warm-Up Transition Time Effects

3. Half-Time Re-Warm-Up

4. Developed Warm-Up Programs

Warm-up efficiency is generally analyzed by explosive effort evaluation. With the emerging engineering technologies, sports analysts begin to further expand their knowledge towards in a new direction. Modified version global positioning system (Gps) implementation for sport sciences is recently used for tracking the displacement and angular movement of players. With the help of this system warm-up and training outcomes can be more easily determined and evaluated. There are still restrictions using Gps in competitions, yet still in many team sports like football, basketball, Gps is used for tracking movements of players.

\section{Stress evaluation}

Stress and anxiety are under investigation nearly in for all research areas. People may face stress and anxiety in working environments, while driving vehicles, studying exams, in a personal affair or even making a presentation in front of a crowded hall. Studies show that a high amount of stress has a negative effect on performance. Determining whether a person is under stress is not an easy task. Questionnaires are the most popular method for answering this question however, people tend to hide their anxiety in many situations. Generally, if there is a possibility that they will be given a punishment, or they are under evaluation. Considering the human body, sympathetic nervous system (SNS) causes changes in skin conductivity, heart rate and saliva production. Advances in sensor technologies in electronics and medical applications [12], make sensitive and highly accurate electro-physiological signal recordings from the human body. Electrodermal activity (EDA) (or galvanic skin response (GSR)), pulse, electrocardiography (ECG), heart rate (HR), respiration recordings are generally used for stress detection studies. Several studies have reported the detection of stress via measurement of physiological signals [13-15]. EDA is the most popular signal used in most studies as skin conductance changes significantly under stress. There are also other studies investigating skin temperature (ST), blood-volume pulse (BVP) and heart rate have also been used to detect stress. Following the signal recording, generating regression models or implementing classification algorithms may determine stress levels. Support vector machine (SVM), artificial neural network (ANN), k-nearest neighbour classifier (KNN) and decision trees (DT) are commonly known machine learning algorithms.

\section{Psychological skills training (PST)}

The importance of PST in the development of athletic performance is widely recognized. REF has a review of PST in elite sports, with a special focus on high-intensity sports (HIS). Birrer et. al comment on their survey that there is a lack of convincing evidence between psychological skills and performance enhancement in HIS [16]. Authors also added that PST should initiate small improvements or progress and it's objective should be improving both the training quality and the competitive performance.

\section{Risk of injury}

Injuries are arguably the primary risk factor for both elite and potential elite players. Missing proper training and competition prevents performance improvement of players and possibly an obstacle in between a long and high level Professional career. For this reason, many studies examine causes of injury and risk parameters. Low energy availability, defined as a relative energy deficiency, may increase the risk of bone stress injuries $[17,18]$. Bone stress injuries resulting of low energy availability underline the dangers of excessive amount of training and competition, especially when combined with inadequate recovery $[18,19]$.

\section{Technological advances}

In the last decade, technology has begun to contribute our daily life even more. Thus, it is obvious that emerging devices are also used in sports. By Goal line technology in football, hawk eye technology in tennis, Gps devices, wearable sensor systems, video analysis, sports clothing design technological advances are getting more involved in every field of different sports. Especially for athletes and swimmers high budget projects try to provide comfort as well as additional performance. In these studies, shoe properties such as mass, midsole compliance, resilience, and longitudinal bending stiffness are observed as in close relation with dissipated energy during running [20-22]. However, achieved energy savings due to running shoe properties are negligibly small [23]. For every $100 \mathrm{~g}$ of added mass per shoe, the energetic cost of running increases by $1.0 \%$, so shoe manufacturers try to design superlight shoes day by day.

\section{Discussion}

Considering the results of available studies in literature, intentions for future studies should focus on limitations that authors mentioned. Yet, current knowledge on warm-up effects are limited. Long-term studies, establishing various warm-up procedures during or off season, may answer correlation between physical performance and physiological, mental or biomechanical variables. By understanding these relations, it can be possible to identify the physiological variable characteristics of desired task performance. Recently this approach has growing interest due to technological advances, as wearable sensor, Gps data. Many Gps device requires personalization which will also help to assess individual difference and offer personalized warm-up or training program development. Small changes detected in physiological signals may result as applying specific warm-up strategies that differ between players. Thus, each player may respond better to the corresponding program provided. Birrer suggest applied sports psychologists should first address the psychological requirements of the sport prior PST program implementations, considering the level of the athlete. As previously discussed, D also underlines the importance of individual differences and PST techniques are not universally applicable without considering these differences. Moreover, there are several methodological variations reported depending on warm-up intensity, duration, recovery time, and type. As Helgerud et. al mentioned [24], it is imperative for team sports practitioners to monitor, external demands and physiological responses of players during training but especially during 
competition. This process has currently been facilitated by timemotion analysis, such as semi-automated camera and statistical systems or by the use of micro-electronics devices). Given their good reliability $[25,26]$, portability, micro-technology devices are now the preferred method of motion-tracking despite their limitation in contact sports caused by collisions, tackling conditions [27-31]. GPS devices provide covered distances, significant sprints or particular movements in discrete zones in the sports field [32]. The outputs of these commercial devices feature triaxial accelerometers sampling at generally $100 \mathrm{~Hz}$ which is more than adequate. Such These embedded accelerometers measure g-force and acceleration in the sum of multiple dimensions as orthogonal axes (anterioposterior (x), mediolateral (y) and vertical (z)) [33]. Accelerometer output then can be used to quantify the change of g-force both in magnitude and direction during accelerating and decelerating movements [30,33-35]. However, estimation metabolic power and energy expenditure of Gps devices and visual tracking systems still do not provide sufficient knowledge and should be explored further in detail.

We comment that electrophysiological signals like EDA, ST, HR should be integrated with Gps devices. HR correlation with oxygen consumption during steady-state submaximal exercise are known as improved blood circulation results as oxygen demand in the muscles. Regression analysis have been used to estimate the energy consumption players [30,36,37]. The main problem of these estimation calculation is that the only available data is obtained in indoor recordings and yet generally during off-season or off competition time interval. The contact nature of many team sports is not possible to take into account. With the integration of wearable sensor in Gps system will significantly contribute the gap in many studies. Not only performance or energy dissipation estimation, as in most sports, the injury rate is greater in competition than practice. Risk indicator determination will also be more accurate with the change of direction in studies mentioned above. Another generalization problem of reported findings in team sports is that independent and single research studies, limit the applicability to alternative team sports or different conditions or group of players.

\section{Conclusion}

We believe obtaining larger datasets and having wider collaborations of different expertise group such as medical doctors, trainer, practitioners, analysts, engineers, statisticians will significantly improve the understanding and analysis of findings. Integration of physiological signals, mental and psychological analysis, muscular, Gps-based electronics systems output combined with the experience of expert trainers may decrease injury risks, enhance performance and improve elite player development. Rather than the integration, investigation of these distinct expertise areas individually does not provide satisfactory outcomes. Current studies support this comment as there are numerous parameters and variables need to be considered and blind approaches ignoring remaining aspects result in contradictory findings.

\section{References}

1. Karageorghis CI, Mouzourides DA, Priest DL, Sasso TA, Morrish DJ, et al. (2009) Psychophysical and ergogenic effects of synchronous music during treadmill walking. J Sport Exerc Psychol 31(1): 18-36.
2. Chtourou H, Chaouachi A, Hammouda O, Chamari K, Souissi N, et al. (2012) Listening to music affects diurnal variation in muscle power output. Int J Sports Med 33(1): 43-47.

3. Eliakim M, Meckel Y, Nemet D, Eliakim A (2007) The effect of music during warm-up on consecutive anaerobic performance in elite adolescent volleyball players. Int J Sports Med 28(4): 321-325.

4. Pujol TJ, Langenfeld ME (1999) Influence of music on Wingate anaerobic test performance. Percept Mot Skills 88(1): 292-296.

5. Bishop D (2003) Warm up I: potential mechanisms and the effects of passive warm up on exercise performance. Sports Med 33(6): 439-454.

6. McGowan CJ, Pyne DB, Thompson KG, Rattray B (2015) Warm-up strategies for sport and exercise: mechanisms and applications. Sports Med 45(11): 1523-1546.

7. Racinais S, Oksa J (2010) Temperature and neuromuscular function. Scand J Med Sci Sports 20(3): 1-18.

8. Fradkin AJ, Zazryn TR, Smoliga JM (2010) Effects of warming-up on physical performance: a systematic review with meta-analysis. J Strength Cond Res 24(1): 140-148.

9. Silva LM, Neiva HP, Marques MC, Izquierdo M, Marinho DA, et al. (2018) Effects of Warm-Up, Post-Warm-Up, and Re-Warm-Up Strategies on Explosive Efforts in Team Sports: A Systematic Review. Sports Med 48(10): 2285-2299.

10. Edge J, Bishop D, Goodman C, Dawson B (2005) Effects of high and moderate-intensity training on metabolism and repeated sprints. Med Sci Sports Exerc 37(11): 1975-1982.

11. Marques MC, Izquierdo M (2014) Kinetic and kinematic associations between vertical jump performance and 10-m sprint time. J Strength Cond Res 28(8): 2366-2371.

12. Sevil M, Hajizadeh I, Samadi S, Feng J, Lazaro C, et al. (2017) Social and competition stress detection with wristband physiological signals. IEEE 14th International Conference on Wearable and Implantable Body Sensor Networks (BSN), Eindhoven, Netherlands.

13. Uzun A, Ozdemir G, Erogul O (2018) Correlation of Facial Expressions and Physiological Signals in Mental Stress Estimation. World Congress on Medical Physics and Biomedical Engineering, IUPESM, Prague, Czech Republic.

14. OM Mozos, V Sandulescu, S Andrews, D Ellis, Nicola B, et al. (2017) Stress Detection Using Wearable Physiological and Sociometric Sensors. International Journal of Neural Systems 27(2): 1-17.

15. F Mokhayeri, MR Akbarzadeh T, S Toosizadeh (2011) Mental Stress Detection Using Physiological Signals Based on Soft Computing Techniques. 18th Iranian Conference of Biomedical Engineering, ICBME 232-237.

16. Birrer D, Morgan G (2010) Psychological skills training as a way to enhance an athlete's performance in high-intensity sports. Scand J Med Sci Sports 20(2): 78-87.

17. Tenforde AS, Barrack MT, Nattiv A, Fredericson M (2016) Parallels with the female athlete triad in male athletes. Sports Med 46(2): 171-182.

18. De Souza MJ, Nattiv A, Joy E, Misra M, Williams NI, et al. (2014) Female Athlete Triad Coalition consensus statement on treatment and return to play of the female athlete triad. Curr Sports Med Rep 13(4): 219-232.

19. Barrack MT, Gibbs JC, De Souza MJ, Williams NI, Nichols JF, et al. (2014) Higher incidence of bone stress injuries with increasing female athlete triad-related risk factors: a prospective multisite study of exercising girls and women. Am J Sports Med 42(4): 949-958.

20. Hoogkamer W, Kipp S, Frank JH, Farina EM, Luo G, et al. (2018) A Comparison of the Energetic Cost of Running in Marathon Racing Shoes. Sports Med 48(4): 1009-1019.

21. Worobets JT, Wannop JW, Tomaras E, Stefanyshyn D (2014) Softer and more resilient running shoe cushioning properties enhance running economy. Footwear Sci 6(3): 147-153.

22. Roy JP, Stefanyshyn DJ (2006) Shoe midsole longitudinal bending stiffness and running economy, joint energy, and EMG. Med Sci Sports Exerc 38(3): 562-569. 
23. Fuller JT, Bellenger CR, Thewlis D, Tsiros MD, Buckley JD, et al. (2015) The effect of footwear on running performance and running economy in distance runners. Sports Med 45(3): 411-422.

24. Helgerud J, Engen LC, Wisloff U (2001) Aerobic endurance training improves soccer performance. Med Sci Sports Exerc 33(11): 1925-1931.

25. Gray AJ, Jenkins D, Andrews MH, Taaffe DR, Glover ML (2010) Validity and reliability of GPS for measuring distance travelled in field-based team sports. J Sport Sci 28(12): 1319-1325.

26. Gabbett TJ, Jenkins DG, Abernethy B (2012) Physical demands of professional rugby league training and competition using microtechnology. J Sci Med Sport 15(1): 80-86.

27. Coutts AJ, Kempton T, Sullivan C, Bilsborough J, Cordy J, et al. (2015) Metabolic power and energetic costs of professional Australian football match-play. J Sci Med Sport 18(2): 219-224.

28. Fox R, Patterson SD, Waldron M (2017) The relationship between heart rate recovery and temporary fatigue of kinematic and energetic indices among soccer players. Sci Med Football 1(2): 132-138.

29. Sirotic AC, Coutts AJ, Knowles H, Catterick C (2009) A comparison of match demands between elite and semi-elite rugby league competition. J Sport Sci 27(3): 203-211.

30. Austin D, Gabbett T, Jenkins T (2011) Tackling in professional rugby league. J Strength Cond Res 25 (6): 1659-1663.
31. Higham DG, Pyne DB, Anson JM, Eddy A (2012) Movement patterns in rugby sevens: effects of tournament level, fatigue and substitute players. J Sci Med Sport 15(3): 277-282.

32. Aughey RJ (2010) Australian football player work rate: evidence of fatigue and pacing? Int J Sports Physiol Perform 5(3): 394-405.

33. Varley MC, Fairweather I, Aughey RJ (2012) Validity and reliability of GPS for measuring instantaneous velocity during acceleration, deceleration, and constant motion. J Sport Sci 30(2): 121-127.

34. McLellan CP, Lovell DI (2012) Neuromuscular responses to impact and collision during elite rugby league match play. J Strength Cond Res 26(5): 1431-1440.

35. Lovell TW, Sirotic AC, Impellizzeri FM, Coutts AJ (2013) Factors affecting perception of effort (session rating of perceived exertion) during rugby league training. Int J Sports Physiol Perform 8(1): 62-69.

36. Garby L, Astrup A (1987) The relationship between the respiratory quotient and the energy equivalent of oxygen during simultaneous glucose and lipid oxidation and lipogenesis. Acta Physiologica Scandinavica 129(3): 443-444.

37. Waldron M, Highton J, Daniels M, Twist C (2013) Preliminary evidence of transient fatigue and pacing during interchanges in rugby league. Int J Sport Physiol Perform 8(2): 157-164 